\title{
USO DO SUBGALATO DE BISMUTO PARA HEMOSTASIA LOCAL EM HEPATECTOMIAS PARCIAIS EM RATOS
}

\author{
BISMUTH SUBGALLATE FOR LOCAL HEMOSTASIS AFTER PARTIAL \\ HEPATECTOMY IN RATS
}

\author{
Paulo Cesar Arroyo Júnior, ACBC-SP ${ }^{1}$ \\ Rita de Cássia Martins Alves da Silva ${ }^{2}$ \\ Dalisio de Santi Neto ${ }^{3}$ \\ Devair de Santana Júnior ${ }^{4}$ \\ Fabrício Dominici Ferreira ${ }^{5}$ \\ Renato Ferreira da Silva, TCBC-SP ${ }^{6}$
}

\begin{abstract}
RESUMO: Objetivo: Avaliar os efeitos do subgalato de bismuto (SGB) usado na área cruenta pós-hepatectomia parcial, quanto a sangramento, aderências e estudo histológico. Método: Foram utilizados 30 ratos divididos em dois grupos iguais,submetidos à hepatectomias parciais com bisturi de lâmina. Para realizar a hemostasia no grupo 1 (G1), foi utilizado bisturi eletrônico e no grupo 2 (G2), SGB. No $7^{\circ}$ dia de pós-operatório (PO), os animais foram mortos, e na cavidade abdominal foram observados sangramento, aderências e, a seguir, realizada a hepatectomia total englobando todos os tecidos adjacentes para análise histológica. No estudo histológico foram analisados: trombose da microcirculação, reação granulomatosa, necrose, fibrose, grau de inflamação e aderências. Resultados: Não foi observado sangramento no PO nos dois grupos. No G1 estavam presentes aderências de omento ao fígado, consideradas neste trabalho como fisiológicas em $80 \%$ dos ratos, e no G2 estas aderências foram por outros órgãos, consideradas neste trabalho como anômalas em todos os casos. No exame histológico, quanto à reação granulomatosa e aderências, todos os ratos as apresentaram. Quanto à trombose e necrose o G1 apresentou maior intensidade. Quanto à fibrose e grau de inflamação os resultados foram semelhantes em ambos os grupos. Conclusões: Ambos os métodos são eficientes para prevenir hemorragia. O G2 apresentou aderências anômalas inviabilizando seu uso em humanos. O G1 revelou mais trombose e necrose. Quanto à reação granulomatosa, fibrose, grau de inflamação e aderências microscópicas, os resultados foram iguais nos dois grupos.
\end{abstract}

Descritores: Hepatectomia; Hemostasia cirúrgica; Bismuto; Aderências; Cirurgia do sistema digestivo; Perda sangüínea cirúrgica; Hemorragia pós-operatória.

1. Cirurgião Geral, $1^{\circ}$ Assistente da Unidade de Cirurgia e Transplante de Fígado do Hospital de Base (FUNFARME) de São José do Rio Preto,SP; Mestrando do curso de pós graduação em Medicina Interna da Faculdade de Medicina de São José do Rio Preto (FAMERP).

2. Professora Adjunta do Departamento de Medicina Integrada da Faculdade de Medicina de São José do Rio Preto (FAMERP).; Doutora em Medicina pela UNICAMP; Gastroenterologista e Hepatologista Responsável pela Unidade de Transplante de Fígado do Hospital de Base (FUNFARME) de São José do Rio Preto,SP.; Pós-Doutorado na Universidade de Birmingham, Inglaterra.

3. Especialista em hepatopatologia na Unidade de Transplante de Fígado do Hospital de Base (FUNFARME) de São José do Rio Preto,SP; Membro do Departamento de Patologia e Medicina Legal da Faculdade de Medicina de São José do Rio Preto (FAMERP).

4. Médico Residente do Departamento de Ortopedia da Faculdade de Medicina de São José do Rio Preto,SP (FAMERP).

5. Médico Residente do Departamento de Cirurgia Geral da Faculdade de Medicina de São José do Rio Preto,SP (FAMERP).

6. Professor Adjunto do Departamento de Cirurgia da Faculdade de Medicina de São José do Rio Preto (FAMERP).; Mestre e Doutor em Cirurgia pela UNICAMP; Chefe do Unidade de Cirurgia e Transplante de Fígado do Hospital de Base (FUNFARME) de São José do Rio Preto,SP; Fellow da Unidade de Transplante de Fígado da Universidade de Birmingham, Inglaterra.

Recebido em 07/11/2002

Aceito para publicação em 03/06/2003

Trabalho realizado para a obtenção do Título de Mestre em Medicina Interna pela Faculdade de Medicina de São José do Rio Preto (FUNFARME). 


\section{INTRODUÇÃO}

A hepatectomia é uma cirurgia utilizada para ressecções de tumores primários e secundários ${ }^{1}$, na redução do fígado para transplante em pacientes pediátricos ${ }^{2}$, para dividir o fígado em dois enxertos ${ }^{3} \mathrm{e}$, mais recentemente, utilizada no transplante de fígado intervivos ${ }^{4}$. A divisão anatômica do fígado, proposta por Couinaud ${ }^{5}$, e os avanços tecnológicos, tais como, bisturis de argônio e ultra-sônico ${ }^{6,7}$, "Cell Saver" ultra-som intraoperatório ${ }^{6}$, também contribuíram para o desenvolvimento da cirurgia hepática. Contudo, a hemorragia persiste como a principal complicação nas hepatectomias, alcançando a prevalência de até $40 \%{ }^{7,9}$.

O desenvolvimento das colas biológicas ${ }^{10}$ que são utilizadas na face cruenta do fígado póshepatectomia, diminuem a taxa de hemorragia no pósoperatório. Elas podem apresentar as seguintes complicações: hipotensão arterial, anafilaxia, morte ${ }^{11} \mathrm{e}$ também têm seu uso limitado devido ao alto custo.

$\mathrm{O}$ subgalato de bismuto $\left(\mathrm{C}_{7} \mathrm{H}_{5} \mathrm{BiO}_{6}\right)$ é um sal derivado de um metal pesado, relativamente insolúvel e muito pouco absorvido, com um forte poder adstringente. Thorisdottir et al. ${ }^{12}$, em 1987, concluiram que o fator 12 (Hageman) é ativado pelo subgalato de bismuto (SGB) que acelera a via intrínseca da cascata de coagulação. Em 1989, Maniglia ${ }^{13}$ demonstrou o efeito hemostático do SGB em adenotonsilectomia.

Levando-se em conta que a hemorragia persiste como principal complicação nas cirurgias hepáticas $^{7,9}$, realizamos experimento em ratos utilizando o SGB nas resecções do fígado . O objetivo deste trabalho foi observar os efeitos deste fármaco usado na área cruenta do fígado pós-hepatectomia quanto ao sangramento imediato e tardio, presença de aderências e estudo histológico do fígado remanescente.

Este trabalho foi autorizado pela Comissão de Ética na Experimentação Animal (CEEA) da Faculdade de Medicina de São José do Rio Preto (FAMERP), SP, conforme processo número 002955/ 2002.

\section{MÉTODO}

O estudo foi realizado no biotério da Faculdade de Medicina de São José do Rio Preto (FAMERP). Foram utilizados 30 ratos machos da raça Wistar, pesando entre $240 \mathrm{gr}$ e 445 gramas divididos em dois grupos:
Grupo 1 (G 1) - hepatectomia subsegmentar e uso do bisturi eletrônico na face cruenta $(n=15)$.

Grupo 2 (G 2) - hepatectomia subsegmentar e uso de subgalato de bismuto na face cruenta $(\mathrm{n}=15)$.

No período pré-operatório, os animais foram colocados em gaiolas coletivas, com luminosidade moderada, temperatura ambiente entre 25 e $30^{\circ} \mathrm{C}$, submetidos ao mínimo contato humano e mantidos em jejum por um período de 12 horas até o procedimento cirúrgico.

$\mathrm{O}$ anestésico utilizado no procedimento foi o pentobarbital $\left(\mathrm{HYPNOL}^{\circledR}\right)$; cada frasco-ampola contém $50 \mathrm{ml}$ e cada $1 \mathrm{ml}$ contém $30 \mathrm{mg}$ da substância ativa. Foi realizada uma diluição de $3 \mathrm{ml}$ do anestésico (90mg) em $7 \mathrm{ml}$ de solução fisiológica a $0,9 \%$, obtendo-se desta forma $9 \mathrm{mg}$ por $\mathrm{ml}$ de anestésico. Os ratos foram anestesiados após a tricotomia e a antissepsia, por via peritoneal no quadrante inferior esquerdo do abdômen, com pentobarbital, 30 a 40 $\mathrm{mg} / \mathrm{kg} / \mathrm{dose}^{14}$.

O SGB usado no intra-operatório foi obtido, diluindo-se 5 gramas do pó da substância em $20 \mathrm{ml}$ de solução salina a $0,9 \%$ até se obter uma mistura semelhante a uma pasta ${ }^{13}$. O bisturi eletrônico utilizado foi o WEM Equipamentos, modelo B450, com intensidade de coagulação variando de 3 a 4 Watts (0-10).

Durante o procedimento operatório, o rato foi mantido sob o uso de oxigênio.

A - Procedimentos operatórios comuns nos Grupo 1 e Grupo 2:

1. Anestesia intraperitoneal com pentobarbital (30 a $40 \mathrm{mg} / \mathrm{kg} /$ dose);

2. Imobilização do animal em decúbito dorsal em placa de madeira, fixação dos membros e tricotomia da parede abdominal anterior;

3. Antissepsia da pele com povidine alcoólico;

4. Laparotomia por incisão mediana de no máximo $6 \mathrm{~cm}$ de extensão;

5. Realização de Manobra de Pringle;

6.Abordagem do lobo hepático esquerdo;

7.Secção de fragmento de aproximadamente $2 \mathrm{~cm}$ do lobo lateral esquerdo do fígado por meio de incisão com bisturi de lâmina.

\section{Grupo 1:}

B - Procedimento operatório adicional no

1. Cauterização da área cruenta com bisturi eletrônico até hemostasia completa;

2. Desfeita a manobra de Pringle; 
3. Devolução do fígado à cavidade abdominal;

4. Observação da área cruenta por cinco minutos sob visão direta para revisão da hemostasia, e, se necessário, nova cauterizacão;

5. Na ausência de sangramento, fechamento da cavidade em dois planos: $1^{\circ}$. com fio absorvível Vicryl ${ }^{\circledR} 3.0$ e o $2^{\circ}$ com fio inabsorvível Mononylon ${ }^{\circledR}$ 4.0.

\section{Grupo 2:}

\section{C - Procedimento operatório adicional no}

1. Aplicação da pastas do subgalato de bismuto sobre a área cruenta usando um cotonete limpo;

2. Desfeita a manobra de Pringle;

3. Devolução do fígado à cavidade abdominal;

4. Observação da área cruenta por cinco minutos sob visão direta para revisão da hemostasia e, se necessário, reaplicação da pasta de subgalato de bismuto;

5. Na ausência de sangramento, fechamento da cavidade abdominal em dois planos: $1^{\circ}$. com fio absorvível Vycril@ 3.0 seguido do $2^{\circ}$. com fio inabsorvível Mononylon ${ }^{\circledR} 4.0$.

Durante a recuperação anestésica, todos os ratos foram colocados em um recipiente fechado sob uso de oxigênio. Recuperados do procedimento anestésico, os mesmos foram mantidos sob os cuidados do período pré-operatório com dieta alimentar conforme a necessidade.

Todos os animais foram mortos após sete dias de pós-operatório, obedecendo os seguintes procedimentos:

1. Anestesia intraperitoneal com pentobarbital (30 a $40 \mathrm{mg} / \mathrm{kg} / \mathrm{dose}$ );

2. Imobilização do animal em decúbito dorsal em placa de madeira e fixação dos membros;

3. Antissepsia local com povidine alcoólico;

4. Laparotomia por incisão mediana de no máximo $6 \mathrm{~cm}$ de extensão;

5. Observação minuciosa da cavidade abdominal à procura de sangramento;

6. Observação macroscópica e identificação das aderências ao fígado das estruturas vizinhas;

7. Exsanguinação por punção venosa até a parada cardíaca, através da veia cava inferior no nível da junção das veias ilíacas;

8. Hepatectomia total incluindo as aderências para estudo histológico.
No estudo histológico foram analisados os seguintes aspectos:

1. Trombose da microcirculação próximo à área cruenta observada em presente e ausente;

2. Reação granulomatosa a corpo estranho, observada em presente e ausente;

3. Necrose de lóbulos hepáticos na área cruenta observadas como:

0 - ausente; 1 - focos isolados de necrose; 2 - fragmentos numerosos de necrose; 3 - lóbulos inteiros de necrose.

4. Fibrose da área cruenta observada em:

0 - ausente; 1 - leve; 2 - moderada; 3 - intensa.

5. Grau de inflamação observado em:

0 - ausente; 1 - presença de neutrófilos esparsos; 2 - concentração maior de neutrófilos com raros microabscessos; 3 - numerosos micro-abscessos.

6. Estudo histológico das aderências observadas em presente e ausente.

Foi utilizado o teste de Fisher para comparar a proporção dos eventos observados nos grupos estudados. O teste $\mathrm{t}$ para a comparação das médias e Mann-Whitney para comparar variáveis que não apresentaram distribuição normal. O nível de significância adotado foi de $\mathrm{p}<0,05$.

\section{RESULTADOS}

\section{to e tardio}

A - Observação do sangramento imedia-

Não foi observado sangramento no pós-operatório imediato e tardio em ambos os grupos, após os procedimentos descritos.

\section{B - Observação de aderências}

Foram observadas aderências em ambos os grupos. Ressaltamos a grande quantidade de vísceras aderidas à área cruenta do fígado no $\mathrm{G}$ 2 , como mostra a Tabela 1. Conceituaremos neste experimento como aderência pós-operatória fisiológica, aquela em que o omento fez parte do bloqueio no processo inflamatório e aderência pósoperatória anômala, aquela na qual o omento não participou do bloqueio no processo inflamatório. O G 1 apresentou 12 ratos com aderências pósoperatórias fisiológicas e 3 ratos com aderências pós-operatórias anômalas. No G 2 todos os ratos apresentaram aderências pós-operatórias anômalas. 
Tabela 1 - Número e tipos de órgãos aderidos à superfície cruenta do fígado nos GI e G II.

\begin{tabular}{lccccccc}
\hline & \multicolumn{9}{c}{$\mathbf{N}^{\mathbf{0}}$ de órgãos aderidos } & \multicolumn{1}{c}{ Tipo de órgãos } \\
\hline G 1 & $\mathbf{0}$ & $\mathbf{1}$ & $\mathbf{2}$ & $\mathbf{3}$ & $\mathbf{4}$ & $\mathbf{5}$ & \\
$\mathbf{G}$ & 0 & $12 *$ & 3 & - & - & - & $\begin{array}{l}\text { Omento, parede abdominal, } \\
\text { cólon e duodeno }\end{array}$ \\
& - & 5 & 6 & 2 & - & 2 & $\begin{array}{l}\text { Cólon, estômago, diafragma, } \\
\text { intestino delgado, baço, } \\
\text { parede abdominal, esterno }\end{array}$ \\
\hline
\end{tabular}

* todos constaram de aderência do omento.

**não foi observada aderência do omento neste grupo.

\section{C - Estudo histológico}

Descreveremos abaixo os resultados da análise microscópica em ambos os grupos. Na Tabela 2 estão distribuídos os resultados da observação em relação à presença ou ausência de trombose da microcirculação próxima à área cruenta em ambos os grupos (Figura 1).

Em relação à reação granulomatosa, não tivemos diferença estatística visto que ela estava presente em todos os ratos de ambos os grupos.

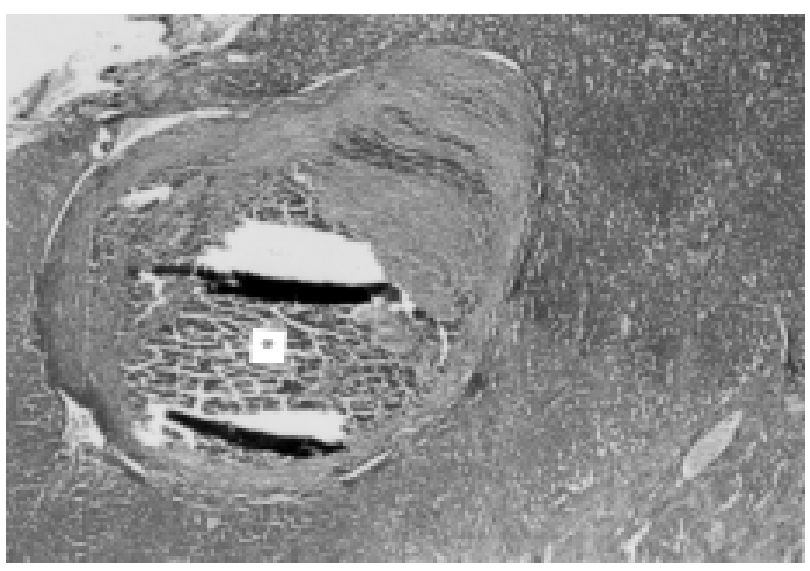

Figura 1 - Trombose de veia centrolobular no GII (*). Tricrômico de Masson, $100 x$.
Para facilitar a análise estatística dos resultados dos graus de intensidade no que se refere à necrose, fibrose e inflamação, os mesmos foram agrupados e distribuídos em dois grupos, considerando-se a intensidade de 0 a 1 como ausente/leve e 2 a 3 como moderada/grave.

$\mathrm{Na}$ Tabela 2 estão distribuídos os resultados da observação que analisa à intensidade de necrose em ambos os grupos (Figura 2).

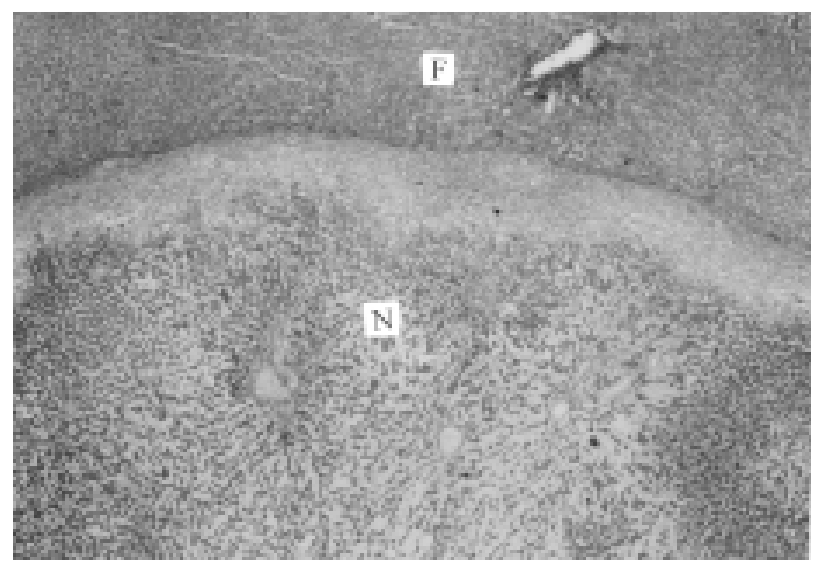

Figura 2 - Necrose extensa $(N)$ panlobular adjacente ao fígado normal (F) no GI. Tricrômico de Masson, $40 x$.

Tabela 2 - Resultados da distribuição de trombose da microcirculação próxima à área cruenta a necrose nos $G$ I e G II.

\begin{tabular}{lcccc}
\hline & \multicolumn{2}{c}{ Trombose $*$} & \multicolumn{2}{c}{ Necrose ** } \\
\hline & Ausente & Presente & Ausente/leve & Moderada/grave \\
\hline G I & 0 & 15 & 0 & 15 \\
G II & 7 & 8 & 9 & 6 \\
\hline$P^{*}=0,0063$ (teste Fisher) & $P^{* *}=0,0007$ (teste Fisher)
\end{tabular}


Quanto à fibrose ( $\mathrm{p}=0,0604)$ (Figura 3) e grau de inflamação $(\mathrm{p}=1,2848)$ não tivemos diferença estatística entre os grupos estudados. O mesmo ocorreu em relação às aderências microscópicas.

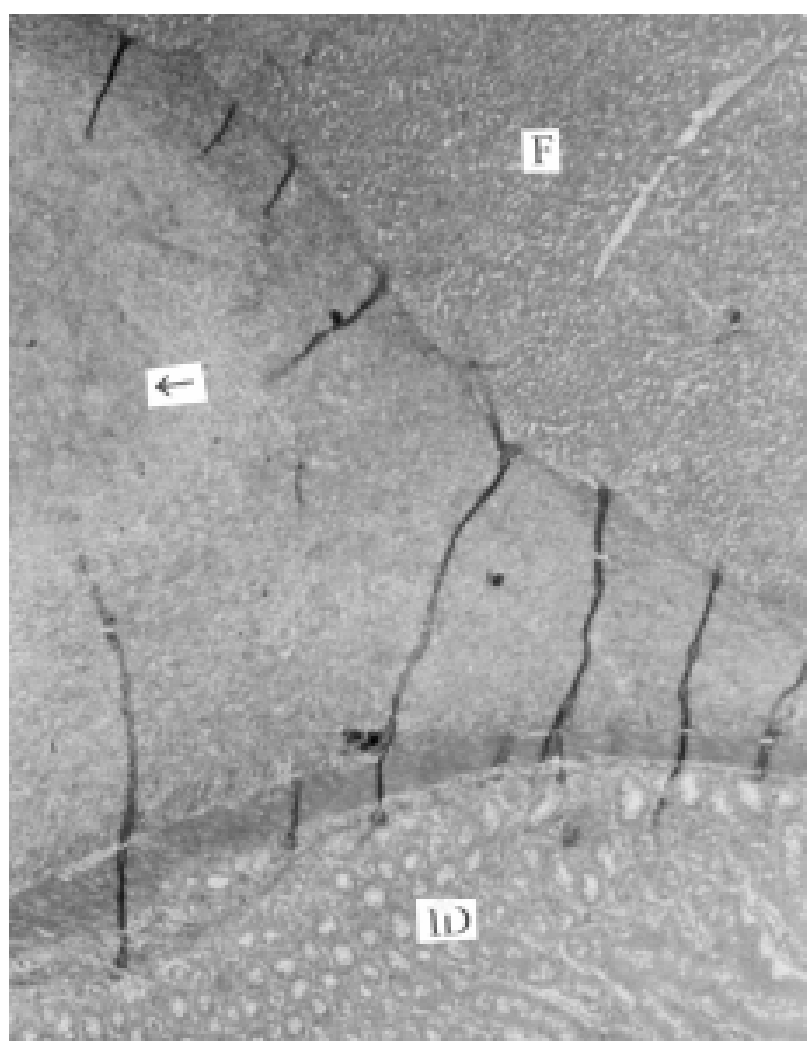

Figura 3 - Fígado $(F)$ e intestino delgado (ID): extensa área de fibrose com reação inflamatória ao subgalato de Bismuto (seta). Tricrômico de Masson, $40 x$.

\section{DISCUSSÃO}

Apesar do progresso na cirurgia hepática, a hemorragia ainda persiste como a causa principal de morbidez ocorrendo em até $40 \%$ das ressecções ${ }^{7,9}$. A utilização do subgalato de bismuto neste experimento procurou demonstrar que esta substância pode diminuir os índices de sangramento nas hepatectomias, visto que é utilizada para este fim nas adenotonsilectomias ${ }^{13}$. Com a função de ativar o fator 12 (Hageman) e com isto acelerar a via intrínseca da cascata de coagulação ${ }^{12}$, a pasta do subgalato de bismuto quando aplicada à superfície cruenta do fígado, mostrou-se eficiente, não havendo sangramento imediato ou tardio. No entanto, o mesmo resultado ocorreu com o grupo que utilizou o bisturi eletrônico. Este pode ser considerado o primeiro estudo encontrado na literatura, utilizando-se o subgalato de bismuto no fígado para fins de hemostasia.
O que podemos ressaltar à inspeção da cavidade no momento da morte dos animais, foram os tipos e números de aderências em ambos os grupos. No $\mathrm{G} 1$ houve predomínio delas entre o fígado e o omento em doze casos e, em três, o omento aderiu ao fígado em associação com outros órgãos como o duodeno, cólon e parede abdominal. No G 2 não houve coalescência do fígado com o omento, estas ocorreram entre este e outros órgãos, sendo que em dez casos, mais de um órgão aderiu à face cruenta do fígado. Em dois casos, cinco órgãos ficaram aderidos à face cruenta do fígado, demonstrando um grande poder de coalescência como mostrado na Tabela 1 .

Para explicarmos neste trabalho porque o grupo que utilizou SGB não apresentou aderências de omento, é necessário entender o papel do SGB na cascata de coagulação. Este farmaco atua na ativação do fator 12 (Hageman), e isto implica em acelerar a via intrínseca da cascata de coagulação. Este fator ativa o fator 11 - Antecedente da Tromboplastina Plasmática (PTA ${ }^{15}$. O PTA é importante na prevenção da formação de aderências, atuando na matriz do gel de fibrina, transformando-a em produtos os quais não tem ação aderente ${ }^{16}$. É possível que o SGB atue inibindo a fibrinólise e com isto aumente a quantidade de fibrose, tendo como resultado final o aumento de adesão de outros órgãos, por impedir a função natural do omento. Outro fator que pode ter contribuído para o aumento de aderências deve ter sido a presença do SGB como um corpo estranho, pois o mesmo não foi absorvido.

No estudo histológico após analisarmos a presença ou ausência de trombose da microcirculação próxima à área cruenta em ambos os grupos, observamos que no $\mathrm{G} 1$ houve trombose em todos os fígados estudados, enquanto que no $\mathrm{G} 2$, oito fígados apresentaram este evento como mostra a Tabela 2. Este achado na microcirculação próxima à área cruenta é significativamente maior no grupo que utilizou o bisturi eletrônico $(\mathrm{p}=0,0063)$. Este resultado coloca o bisturi eletrônico como melhor opção que o subgalato de bismuto.

No exame histológico, em relação à reação granulomatosa, observou-se que estava presente em todos os ratos de ambos os grupos, não havendo superioridade entre os métodos.

O exame histológico, quanto à necrose, revelou que nos quinze ratos do G1 ela variou de modera$\mathrm{da} /$ grave, enquanto que no $\mathrm{G} 2$ mostrou seis ratos com lesões de moderada/grave e em nove ela era ausen- 
te/leve. A necrose na superfície cruenta foi significativamente maior no grupo com uso do bisturi eletrônico $(\mathrm{p}=0,0007)$. Isto seria teoricamente uma desvantagem no G1, pois o dano maior pode favorecer as infecções em até $50 \%$ dos $\operatorname{casos}^{17}$.

A análise histológica, quanto à fibrose, revelou que nos quinze ratos do $\mathrm{G} 1$ nove apresentaram fibrose ausente/leve e seis moderada/grave, enquanto que o $\mathrm{G} 2$ apresentou três ratos com lesão ausente/leve e doze com lesão moderada/grave. Embora a fibrose mais intensa tivesse ocorrido no $\mathrm{G}$ 2 , não houve significância estatística $(\mathrm{p}=0,0604)$. $\mathrm{O}$ fato do $\mathrm{G} 2$ apresentar mais fibrose provavelmente corrobora os achados sobre a presença de mais aderências.

Quanto ao grau de inflamação, tanto o $\mathrm{G} 1$ como o $\mathrm{G} 2$ apresentaram os mesmos resultados: sete ratos com ausente/leve e oito com moderada/grave. O processo inflamatório foi idêntico em ambos os gru- pos, não tendo, portanto, significância estatística, apesar da necrose ser mais intensa no $\mathrm{G} 1$.

No que se refere às aderências microscópicas na superfície cruenta do fígado, elas ocorreram em ambos os grupos, e em todos os animais estudados.

Após a análise do presente estudo podemos concluir que:

1. Ambos os métodos foram eficientes para prevenir hemorragia;

2. O grupo do subgalato de bismuto produziu aderências pós-operatórias patológicas, o que pode inviabilizar o seu uso em humanos;

3. O grupo do bisturi eletrônico foi mais eficiente em provocar trombose na microcirculação;

4. O grupo do subgalato de bismuto apresentou menos necrose;

5. Quanto à reação granulomatosa, fibrose, grau de inflamação e aderências microscópicas, os resultados em ambos os grupos foram iguais.

\begin{abstract}
Background: This study aimed to observe the effects of bismuth subgallate used on bleeding surfaces after hepatectomy, in relation to bleeding, adhesions and histological study. Methods: A total of 30 rats divided in 2 equal groups were studied. Partial hepatectomy was performed in both groups. In group $1(G-1)$ an electric scalpel was used and in group $2(G-2)$ bismuth subgallate for hemostasis of the bloody surface was aplied. On the $7^{\text {th }}$ post-operative day, rats were sacrificed and the abdominal cavity was investigated with respect to bleeding and adhesions. A total hepatectomy with the adhesions was performed for histological study. In the histological study, microcirculation thrombosis, granulomatosis reaction, necrosis, fibrosis, degree of inflammation and adhesions were analyzed. Results: There was no post-operative bleeding in both groups. Adhesions were observed on all rats in both groups. G-1 presented omentum adhesions to the liver, here denominated physiological in $80 \%$ of the rats and the G-2 presented adhesions of other organs, here named pathological in all cases. The histological study showed that all rats presented a granulomatosis reaction and adhesions. G-1 presented a greater intensity of thrombosis and necrosis. There was no significant difference between the two groups in relation to fibrosis or the degree of inflammation. Conclusions: This study demonstrated that both methods were efficient to prevent hemorrhage. G-2 presented pathological adhesions suggesting its use is unfeasible in human beings. G-1 presented more thrombosis and also more necrosis. The granulomatosis reaction, fibrosis, degree of inflammation and microscopic adhesions were similar in between groups.
\end{abstract}

Key Words: Hepatectomy; Hemostasis, surgical; Bismuth; Adhesions; Digestive system surgery; Blood loss, surgical; Postoperative hemorrhage.

\section{REFERÊNCIAS}

1. Buell JF, Rosen S, Yoshida A, et al. - Hepatic resection: effective treatment for primary and secondary tumors. Surgery, 2000,128(4):686-693.

2. de Hemptinne B, Salizzoni M, Yandza, et al. - Indication, technique and results of liver graft volume reduction before orthotopic transplantation in children. Transplant Proc, 1987,19(5):3549-3551.
3. Bismuth H, Morino M, Castaing D, et al. - Emergency orthotopic liver transplantation in two patients using one donor liver. Br J Surg, 1989, 76(7):722-724.

4. Raia S, Nery JR, Mies S - Liver transplantation from liver donors. Lancet, 1989, 2(8661):497.

5. Bismuth $\mathrm{H}$ - Surgical anatomy and anatomical surgery of the liver. World J Surg, 1982, 6(1):3-9.

6. Little JM, Hollands MJ - Impact of the CUSA and operative ultrasound on hepatic resection. HPB Surg, 1991,3(4):271-278. 
7. Blumgart LH - "Liver resection - liver and biliary tumours". In Blumgart LH (ed) - Surgery of the liver and biliary tract. New York. Churchill Livingstone, 1994, pp. 1495-1538.

8. Zantut LFC, Zantut PEC, Birolini D - Laparoscopia e autotransfusão em pacientes traumatizados. Estudo de 21 casos. Rev Col Bras Cir, 1991,18(4):139-142.

9. Silva RF, Silva RCMA - "Metástases hepáticas: abordagem cirúrgica”. In Castro LP, Savassi-Rocha PR, Rodrigues MAG, et al (eds) - Tópicos em gastroenterologia. Câncer do aparelho digestivo. Rio de Janeiro. Medsi, 2002, pp. 443-454.

10. Kram HB, Reuben BI, Fleming AW, et al. - Use of fibrin glue in hepatic trauma. J Trauma, 1988, 28(8):1195-1201.

11. Berguer R, Staerkel RL, Moore EE, et al. - Warning: fatal reaction to the use of fibrin glue in deep hepatic wounds. Case reports. J Trauma, 1991, 31(3):408-411.

12. Thorisdottir H, Ratnoff OD, Maniglia AJ - Activation of Hageman factor (factor XII) by bismuth subgallate, a hemostatic agent. J Lab Clin Med, 1988,112(4):481-486.

13. Maniglia AJ, Kushner H, Cozzi L-Adenotonsillectomy. A safe outpatient procedure. Arch Otolaryngol Head Neck Surg, 1989,115(1):92-94.
14. Waynforth HB, Flecknell PA - "Specific surgical operations". In Waynforth HB, Flecknell PA (eds) Experimental and surgical technique in the rat. $2^{\text {nd }}$ Edition. London. Academic Press, 1992, pp. 203312.

15. Mosher DF - "Distúrbios da coagulação". In Wyngaarden JB, Smith LH (eds) - Cecil Tratado de Medicina Interna. $18^{\mathrm{a}}$ Edição. Rio de Janeiro. Guanabara Koogan, 1990, pp.934-938.

16. Liakakos T, Thomakos N, Fine PM, et al. - Peritoneal adhesions: etiology, pathophysiology, and clinical significance. Recent advances in prevention and management. Dig Surg, 2001, 18(4):260-273.

17. Rokke O, Nesvik I, Sondenaa K - Traumatic and postoperative ischemic liver necrosis: causes, risk factors and treatment. Dig Surg, 2000,17(6):595601.

Endereço para correspondência:

Paulo Cesar Arroyo Júnior

Rua Gilberto Lopes da Silva, 55/603

Jardim Redentor - CEP: 15085-390

São José do Rio Preto - SP 